\title{
Tuned Probehead for NMR Spectroscopy in Magnetically Ordered Materials
}

\author{
B. Sedlák, K. Kouřil, V. Chlan, R. Řezníček, P. KřišŤan, M. Pfefffer, \\ J. ENGLICH AND H. ŠTĚPÁNKOVÁ \\ Charles University in Prague, Faculty of Mathematics and Physics \\ V Holešovičkách 2, 18000, Praha 8, Czech Republic
}

\begin{abstract}
This work describes the design of a tuned NMR probehead that was developed for measurement of very broad spectra in fully impedance matched mode. The probehead is constructed as an insert of continuous flow cryostat to allow operation down to helium temperatures. Properties of the probehead are demonstrated on ${ }^{57} \mathrm{Fe}$ NMR in magnetic oxide with spinel structure.
\end{abstract}

PACS numbers: 07.57.Pt, 76.60.-k, 76.60.Lz

\section{Introduction}

Hyperfine fields acting on atomic nuclei arise due to strong interaction between electron and nuclear magnetic moments. The presence of a strong hyperfine magnetic field in magnetically ordered materials is a fundamental aspect in application of NMR spectroscopy to investigation of these materials, which implies several consequences. From methodological point of view, it is necessary to respect that the NMR spectra are wide in frequency scale and the resonance lines display considerable inhomogeneous broadening when individual line widths in the spectra can vary from few $\mathrm{kHz}$ to tens of $\mathrm{MHz}$. The NMR spectrum is then usually obtained in a frequency-swept regime as an envelope of individual Fourier transform of signals measured at different excitation frequencies.

Experiments performed on samples that require high intensity of radiofrequency (rf) pulses or exhibit weak NMR signals require the use of tuned probehead where the rf coil is part of the resonance circuit. Tuning of the resonance circuit is achieved by a variable capacitor. For relatively narrow spectra the impedance matching in such a probehead can be set manually prior to the experiment, using either inductive or capacitive components, and remains hardwired. If desired frequency or temperature range is wider, then such an approach is no longer viable.

The next step in improving probehead circuit is two-capacitor concept, which allows for both setting the resonance frequency and matching the probehead impedance to the transmitter and receiver line. Serious limitations to probehead construction come from the fact that it is often needed to measure temperature dependence of NMR spectra. Helium flow cryostats are commonly used for this purpose, and therefore, the space for the probehead is limited by a narrow bore of the cryostat. Also, operation and control of the variable capacitors placed in a cryostat becomes complicated.
In this paper we describe alternative configuration that conforms to the space requirements while keeping the advantage of the two-capacitor concept. In our probehead, only the tuning capacitor of variable capacitance is positioned in the confined sample space, together with excitation/detection coil of fixed inductance $L$ and series resistance $R_{\mathrm{S}}$, while the matching variable capacitor is placed outside the cryostat. Inevitable consequence of this arrangement is that long enough coaxial line has to be used for connection of the matching capacitor with the rest of the probehead rf circuit. The probehead is intended for the frequency range of $10-100 \mathrm{MHz}$ and the length of the cryostat is $\approx 50 \mathrm{~cm}$; thus for purposes of the circuit analysis it is credible to substitute the cable only by its capacitance.

\section{Probehead design}

Impedance matching of the resonant circuit to the spectrometer, which is represented by impedance $R_{Z}=$ $50 \Omega$ in Fig. 1, can be achieved either by coupling the circuit to the spectrometer by series matching capacitor $C_{V}$ (Fig. 1a), or by connecting the spectrometer to the mid-point of the capacitive divider formed by matching capacitor $C_{1}$ and tuning capacitor $C_{L}$ (Fig. 1b).

Detailed analysis shows that in the given frequency range the circuit configuration in Fig. 1a requires too low values of matching capacitance $C_{V}$ (unattainable in technical practice) [1]. On the other hand, the configuration as shown in Fig. 1b yields achievable values of matching capacitances $C_{1}$.

Capacitances $C_{1}$ and $C_{L}$ for optimal tuning and matching of the circuit at a frequency $f$ can be calculated by using the following formulae:

$$
\begin{aligned}
& C_{1}=\frac{1}{\omega R_{Z}} \sqrt{\frac{R_{Z}}{R_{\mathrm{S}}}-1}=\frac{1}{\omega R_{Z}} \sqrt{\frac{R_{Z} Q}{\omega L}-1}, \\
& C_{L}=\frac{1}{\omega\left(\omega L-\sqrt{R_{\mathrm{S}}\left(R_{Z}-R_{\mathrm{S}}\right)}\right)}
\end{aligned}
$$




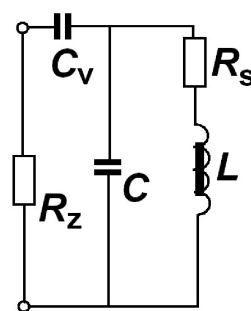

a)

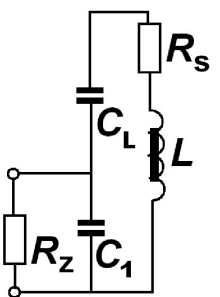

b)
Fig. 1. (a) Matching capacitor $C_{V}$ connected in series with the impedance $R_{Z}$, (b) matching capacitor $C_{1}$ connected in parallel with the impedance $R_{Z}$.

$$
=\frac{1}{\omega\left[\omega L-\sqrt{\frac{\omega L}{Q}\left(R_{Z}-\frac{\omega L}{Q}\right)}\right]},
$$

where $\omega=2 \pi f$ is an angular frequency and $Q=\omega L / R_{\mathrm{S}}$ stands for a quality factor [2]. Numerical examples for $L=0.5 \mu \mathrm{H}$ are provided in Table.

TABLE

Numerical examples of capacitances $C_{1}$ and $C_{L}$ calculated for $L=0.5 \mu \mathrm{H}$ and several frequency and quality factor values.

\begin{tabular}{c|c|c|c|c|c|c|c|c}
\hline \hline & \multicolumn{3}{|c|}{ Frequency $f=50 \mathrm{MHz}$} & \multicolumn{3}{|c}{ Frequency } & \multicolumn{2}{|c}{$f=100 \mathrm{MHz}$} \\
\hline$Q$ & 10 & 30 & 60 & 100 & 10 & 30 & 60 & 100 \\
$C_{1}[\mathrm{pF}]$ & 94 & 186 & 271 & 353 & 24.5 & 61.8 & 93.1 & 122.9 \\
$C_{L}[\mathrm{pF}]$ & 23.8 & 22.5 & 21.8 & 21.5 & 5.49 & 5.42 & 5.33 & 5.27
\end{tabular}

The probehead is able to operate in fully impedance matched mode, in the frequency range of $10-100 \mathrm{MHz}$. It is constructed to operate with modified configuration of Bruker Avance pulse spectrometer and works down to helium temperatures in Oxford Instruments CF 1200 helium flow cryostat. Schematic drawing of the probehead configuration is shown in Fig. 2. Properties of the probehead are demonstrated on ${ }^{57} \mathrm{Fe}$ NMR in magnetic sample.

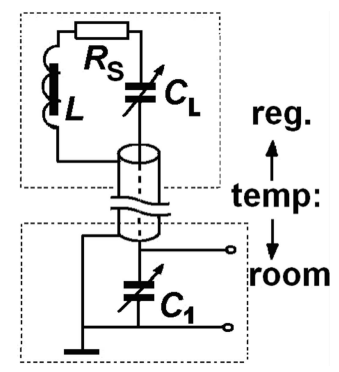

Fig. 2. Probehead configuration.

For resonance frequency tuning, Voltronics NMTM75CEK variable cylindrical glass dielectric capacitor is used (capacitance from below $2 \mathrm{pF}$ to above $75 \mathrm{pF}, \mathrm{DC}$ working voltage $1 \mathrm{kV}, \mathrm{DC}$ withstanding voltage $2 \mathrm{kV}$ ). The impedance matching is set by air dielectric butterfly capacitor of maximal capacitance $30 \mathrm{pF}$, with the possibility to connect additional capacitors via BNC connector. The temperature-regulated part and room temperature part of the probehead are connected by Micro-Coax semi-rigid low temperature cable. Characteristic impedance of the cable is $50 \Omega$, capacitance is $95 \mathrm{pF} / \mathrm{m}$ and voltage withstand is $2300 \mathrm{~V}$.

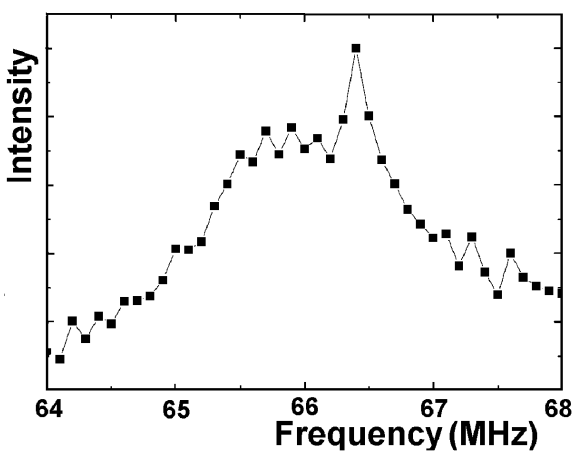

Fig. 3. ${ }^{57} \mathrm{Fe}$ NMR spectrum of $\mathrm{MnFe}_{2} \mathrm{O}_{4}$ in a zero external field at $298 \mathrm{~K}$.

Figure 3 presents ${ }^{57} \mathrm{Fe}$ NMR spectrum of nuclei in $\mathrm{Fe}^{3+}$ ions of octahedral sites in manganese ferrite $\mathrm{MnFe}_{2} \mathrm{O}_{4}$ measured at a temperature of $298 \mathrm{~K}$ in a zero external magnetic field. The spectrum was measured by the CarrPurcell pulse technique. The excitation conditions were adjusted so that only nuclei inside the magnetic domains contributed to the NMR signal. According to our experience with these samples [3], we are convinced that without tuning and impedance matching for every frequency step the spectrum would not be detected at room temperature.

\section{Conclusions}

We designed a NMR probehead that is capable of operation in 10-100 MHz frequency range and allows for tuning and matching of the probehead circuit to the transmitter and receiver line of the spectrometer in the whole range. The design is specific in splitting the probehead circuit into a temperature-regulated part and a room temperature part, which allows usage in narrow bore cryostats without degradation of experiment quality by giving up the possibility of adjustable matching.

\section{Acknowledgments}

This work was supported by the project IAA100100803 of AS CR and by the project MS0021620834 of the Ministry of Education of the Czech Republic. R. ̌r. and P.K. also acknowledge the project SVV-2010-261303.

\section{References}

[1] D.E. MacLaughlin, Rev. Sci. Instrum. 60, 3242 (1989).

[2] J.A. Stratton, Electromagnetic Theory, McGraw-Hill, New York 1941

[3] H. Štěpánková, B. Sedlák, V. Chlan, P. Novák, Z. Simša, Phys. Rev. B 77, 092416 (2008). 